\title{
Upregulation of Pole2 Promotes Clear Cell Renal Cell Carcinoma Progression via AKT/mTOR Pathway and Predicts a Poor Prognosis
}

\section{Yajuan Su}

Tumor Hospital of Harbin Medical University

\section{Changfu Li}

Tumor Hospital of Harbin Medical University

Kun Liu

Tumor Hospital of Harbin Medical University

\section{Liangjun Wei}

Tumor Hospital of Harbin Medical University

\section{Dechao Li}

Tumor Hospital of Harbin Medical University

\section{Wentao Wang}

Tumor Hospital of Harbin Medical University

\section{Yongpeng $\mathrm{Xu}$}

Tumor Hospital of Harbin Medical University

\section{Hongxin Pan}

Tumor Hospital of Harbin Medical University

Lichen Teng ( $\nabla$ tenglichen@2008.sina.com )

Tumor Hospital of Harbin Medical University https://orcid.org/0000-0002-5840-0723

\section{Primary research}

Keywords: renal cell carcinoma, pole2, survival, cell cycle, apoptosis

Posted Date: June 22nd, 2020

DOl: https://doi.org/10.21203/rs.3.rs-35733/v1

License: (c) (i) This work is licensed under a Creative Commons Attribution 4.0 International License. Read Full License 


\section{Abstract}

Background: Pole2 gene is a subunit of DNA polymerases localized in the nucleus, which commonly present in DNA repair. The effect of pole2 in renal cell carcinoma (RCC) still remain unclear. Here we investigate its clinical significance, function in RCC cells and possible mechanism of effect.

Methods: Using TCGA database, we identified that up-regulation of pole2 is associated with poor prognosis in ccRCC. We analyzed association between pole2 expression and T stage or Fuhrman grade. Thus, we investigate the effects of pole2 down-regulation on proliferation, cell cycles, apoptosis and possible mechanism in cells using lentivirus vector with shPole2.

Results: Our study showed overexpression of pole2 in ccRCC samples, compared with normal kidney tissues, moreover, high expression of it related to high Fuhrman grade, also may predict poor prognosis in patients with ccRCC $(p<0.05)$. In cultured cells, knockdown of pole2 would result in inhibit of cell proliferation, increase the apoptosis of cells and arrest cell cycle at $S$ phase. Importantly, knockdown pole2 can lead to down-regulation of p-mTOR and p-AKT expression.

Conclusion: Taken together, our findings suggest that overexpression of pole2 may promote tumorgenesis and progress of ccRCC via AKT/mTOR signaling.

\section{Background}

Renal cell carcinoma (RCC) accounts for approximately $3 \%$ of adult malignancies. ${ }^{1}$ The incidence of RCC increased over the last two decades in all regions of the world and ethnic groups. Kidney cancer also is the $9^{\text {th }}$ most common malignancy and the $14^{\text {th }}$ most common malignancy. ${ }^{2}$ As advancements in imaging detection technique, such as ultrasonography, computed tomography (CT) and MRI, more and more localized kidney cancers were diagnosed, Despite significantly increased detection of RCC, its prognosis varies widely; about $30 \%$ of patients have metastatic disease when diagnosed RCC, and approximately $30 \%$ of patients will develop local or distant metastases after radical or partial nephrectomy. ${ }^{3,4}$ As for organ-confined disease, surgical resection of tumor or kidney is undoubtedly an optimal option, but as for metastatic renal cell cancer (mRCC). Because RCC being highly resistant to chemotherapy and radiation therapy, immunotherapy with cytokine or checkpoit inhibitor only showed lower rate of response for the patients, in the past decade, systemic management has changed significantly, target therapy that mainly inhibit VEGF receptors and mTOR pathway provide many choices for urologists, ${ }^{5}$ however for the most patients, the efficacy in the long-term remains unsatisfactory, so in order to find a new effective target of treatment, further study on the factors that promote to tumorgenesis and metastasis of RCC is still required.

DNA polymerases mainly involve in replication of DNA that can repair DNA damage and correct misincorporation. ${ }^{6}$ DNA polymerase epsilon subunit 2 (POLE2) gene encodes for a 59 kDa protein that play an important role in replication of DNA, including dimerization with POLE1 and affecting C-terminal 
part of the catalytic subunit through protein-protein interactions. Mutation of pole2 is rare, but it can be identified in some diseases condition, such as combined immunodeficiency, colorectal adenomas. ${ }^{7,8}$ However, the exact biological function and clinical significance of pole2 remain unknown.

In this study, we investigated the level of pole2 expression in renal cell carcinoma cells and tissues, moreover, we silenced expression of pole2 with lentivirus-mediated specific shRNA, next we studied the effect of pole2 on the proliferation, apoptosis, and the cell cycle in renal cell carcinoma cells, we also find that AKT/mTOR pathway mediated the effect of pole2. Our study may revealed a new target of therapy for renal cell carcinoma.

\section{Materials And Methods Bioinformatics analysis}

To discover possible clear cell renal cell carcinoma genes, RNA-seq and RNA-seqV2 were downloaded from website of The Genomic Data Commons, containing 506 and 72 paired of ccRCC tissue and normal samples, respectively. All mRNA expression data were normalized. To prevent bias of the samples, we observed the biological coefficient of variation (BCV). The gene expression between cancer and normal tissue samples were compared to identify the differentially expressed gene.

\section{Chemical and antibodies}

Anti-Pole2 was purchased from Sigma (St. Louis, MO, USA). 3-(4,5-dimethylthia- zol-2-yl)-2,5diphenyltetrazolium bromide (MTT) was purchased from Genview (League, TX, USA). Primary antibody against AKT, p-AKT, mTOR and p-mTOR were purchased from Cell Signaling Technology (Beverly, MA, USA). The antibody against GAPDH was obtained from Best ( Nanjing, Jiangsu, P.R.C).

\section{Renal cell carcinoma samples}

fresh matched pairs tissue samples were harvested from 94 patients with renal cell carcinoma who underwent radical nephrectomy or partial nephrectomy between March and August 2017. As for all the patients, written informed consents were obtained, and the protocol was approved by Ethical Committee of Harbin Medical University Cancer Hospital. The cancer tissues and adjacent normal tissues were fixed with $4 \%$ paraformaldehyde for $24 \mathrm{~h}$ and then paraffin-embedded for immunohistochemistry.

\section{Cell culture}

The human renal cell carcinoma cells 7860, and ACHN were obtained from ATCC, and cultured in DMEM medium (Hyclone) that were added with $10 \%$ fetal bovine serum (GIBCO), penicillin $\mathrm{G}(100 \mathrm{U} / \mathrm{ml})$, and 
streptomycin $(100 \mu \mathrm{g} / \mathrm{ml})$ (Sigma-Aldrich). All the cells cultured as a monolayer culture at $37^{\circ} \mathrm{C}$ in a humidified atmosphere containing $5 \% \mathrm{CO}_{2}$.

\section{Lentivrus vectors construction and infection}

The lentiviral vectors were purchased from Shanghai Genechem Company Ltd., China, which composed of the vectors hU6-MCS-CMV-EGFP, and pHelper1.0 and Helper2.0 plasmids. The siRNA sequences targeting pole2 gene were 5' -CCTATTTCCCATGATTCCTTCATA-3' and 5'-GTAATACGGTTATCCACGCG-3' shPOLE2). A non-silencing siRNA (5'- TTCTCCGAACGTGTCACGT -3') was used as the negative control (shCrl). These plasmids were respectively cloned into above vectors. All the cells were seed into a six-well plates at $5 \times 10^{4}$ cells per well and incubated, respectively. Appropriate volumes of lentivirus were added to the cells according to the recommendation of manufacture, when cell fusion reached $70 \%$.

\section{Determination of knockdown rate}

Quantitative real-time PCR (qRT-PCR) was used to examine knockdown rate of pole2 mRNA in cells infected lentivirus vectors. GAPDH gene was used as an internal control gene in qRT-PCR. The examinations were performed in triplicate per sample.

\section{Immunohistochenmistry}

The protein expression patterns of Pole2 were analyzed in 35 renal cell carcinoma tissues, and paired adjacent noncancerous tissues, and 100 formalin-fixed, paraffin-embedded renal cancer tissue samples that obtained from patients who underwent operation between January 2013 and December 2015. According to a two-step protocol, rabbit polyclonal anti-human pole2 antibodies (1:200; Sigma) and visualized with secondary antibody (1:200; Beyotime) were used for staining in the sections $(5 \mu \mathrm{m})$. Positive cells were quantitatively calculated using Image-Pro Plus 6.0.

\section{Western blot analysis}

Whole-cell lysates were obtained as previous described, ${ }^{9} 35 \mu \mathrm{g}$ of which separated on a SDS-PAGE gel, next, they were transferred to a nitrocellulose membrane and incubated with anti-human Pole2 (1:1000), AKT (1:1000), p-AKT (1:1000), mTOR (1:1000), p-mTOR (1:1000) and GAPDH (1:1000) overnight at $4{ }^{\circ} \mathrm{C}$. The following day, the membranes were washed by trisbuffered saline and Tween-20 and incubated with a secondary anti-rabbit IgG antibody conjugated with horseradish peroxidase. Next, membranes were incubated with enhanced chemoluminescence and protein expression was visualized after exposure to $X$ ray film. GAPDH was used as internal control. 


\section{Cell proliferation assay}

7860 and ACHN cells, and the cells infected with pole2-shRNA lentivirus or NC lentivirus were collected, and rypsin-digested, when logarithmic growth phase presented, next, the cells resuspended in standard medium, and then seeded into $96-$ well plates at a density of 2,000 cells/well. The number of GFP fluorescence-positive cells was counted using a Celigo Cell Counting (Nexcelom) on five consecutive days. In addition, $20 \mu \mathrm{L}$ of MTT ( $5 \mathrm{mg} / \mathrm{mL}$ in PBS) was added to each well of the plates to form formazan crystals by metabolically active cells, which were subsequently incubated in the incubator humidified with $\mathrm{CO}_{2}(5 \%)$ for $4 \mathrm{~h}$ at $37 \circ \mathrm{C}$. Later, $100 \mu \mathrm{L}$ of DMSO solution was added following removal of the MTT medium. The absorbance was measured at a wavelength of $490 \mathrm{~nm}$ with microplate reader (Tecan infinite). Triplicate experiments were done.

\section{Cell cycle analysis}

Above mentioned cells were washed twice with ice-cold phosphatebuffered saline (PBS), and fixed with $70 \%$ ice-cold ethanol for $1 \mathrm{~h}$. The cells were washed with D-hank saline, then the cells were stained for $1 \mathrm{~h}$ with propidium iodide (Sigma-Aldrich, P4170) containing $10 \mathrm{mg} / \mathrm{ml}$ RNase (Fermentas EN0531). Finally, the samples were analyzed using a flow cytometer (FACSCalibur, Becton Dickinson).

\section{Cell apoptosis analysis}

Above mentioned cells were washed twice with ice-cold phosphatebuffered saline (PBS), and were stained with Annexin V-APC (ebioscience, 88-8007) following manufacturer's instructions and detected by a flow cytometer (FACS Calibur, Becton Dickinson).

\section{Caspase $3 / 7$ analysis}

The cells were seeded into 96 -well plates, and were cultured at $37^{\circ} \mathrm{C}$ in a humidified atmosphere containing $5 \% \mathrm{CO}_{2}$ for $3-5$ days, then $100 \mu \mathrm{L}$ per well Caspase-Glo solution was added to the new 96 -well plates that containing $1 \times 10^{4}$ cells per well, which next were incubated at room temperature for $1-2 \mathrm{~h}$. The intensity of signal was determined by Microplate Reader (Tecan infinite; M2009PR)

\section{MTT assay}

Cells $\left(2 \times 10^{3}\right)$ were seeded out in 96-well plates (Cornning) in a volume of $100 \mu \mathrm{L}$ and cultivated for five days. Untreated cells were used as a negative control and cells infected with NC were used as a positive control. After this cultivation period, cells were washed once with PBS. Since second day of cultivation, the cells were incubated in $20 \mu \mathrm{L} /$ well $10 \%$ MTT solution $(5.0 \mathrm{mg} / \mathrm{mL}$ in PBS) in the medium for $4 \mathrm{~h}$. SDS 
solution (1.0 g SDS in $10 \mathrm{~mL} 0.01 \mathrm{M} \mathrm{HCl}$ ) was added to each well to release the purple-colored salt from the cells. After $24 \mathrm{~h}$ of incubation, UV-Vis absorption was measured at $490 \mathrm{~nm}$ as the reference wavelength using a microplate reader (Tecan infinite M2009PR).

\section{Statistical analysis}

Statistical analysis was performed using the SPSS16.0 software package. Survival curves were plotted using the Kaplan-Meier method. All values in the text and figures are expressed as the mean \pm SD in the study. Chi-square tests and paired Wilcoxon tests were used for evaluating the study results. The association between pole2 expression and patients' clinical-pathologic characteristics was compared using Fishe's exact test. P-value $<0.05$ was considered as statistical significance.

\section{Results}

\section{Pole2 is a highly expressed gene and a poor prognostic factor in ccRCC}

Based on the bioinformatic analysis of TCGA-cCRCC datasets, genes with a fold-change $>2$ were included in our study, finally, we identified pole2 was an optimal candidate gene. Because pole2 expression was 2.585 fold higher in cCRCC tissues than in the normal kidney tissues ( $P=8.70 E-14)$ (Figure 1A and $B$ ). Based on the median value of pole2 mRNA expression level, patients were divided into low-pole2 and high-pole2 groups. Kaplan-Meier analysis showed that high level of pole2 expression can significantly predict poor overall survival in patients with cCRCC and chromophobe kidney cancer with hazard ratio (HR) of 1.4 (95\% Cl: 1.09-1.8) and HR of 2.1 95\% Cl: 1.3-3.39), respectively, $(\mathrm{p}<0.05$, Figure 2A and B), but not in papillary RCC (Figure 2C). Although the result showed that pole2 may be a independent regulator in ccRCC, but its biological function remains unclear. To investigate expression pattern of pole2 in ccRCC, we performed immunohistochemistrical staining with the tissue sections, which included the adjacent non-cancerous tissues and cancerous tissues with different pathological T stages and Fuhrman grades (Figure 2D and 2E). Due to pole2 is mainly localized in cell nucleus, so we quantified pole2-positive cells, and we found that the number of positive cells was higher in the cancerous tissues than in adjacent noncancerous tissues, meanwhile, no significant relationships were identified between pole2 expression and any other clinical features, such as patients' age, gender, and side $(p>0.05)$. There was not a significant difference in any sections of $T$ stage ( $p>0.05)$, on the contrary, number of positive cells in lower Fuhrman grade ( $G 1$ or $G 2)$ were less than that in sections of $G 3$, respectively $(p<0.05)$. However, compared with sections of $\mathrm{G} 4$, we did not find statistical difference in number of positive cells $(p>0.05)$ (Table1).

\section{Expression of pole2 mRNA was determined by real time quantitative PCR in 7860 and ACHN cells}


Abundance of pole2 expression in human renal cancer cell lines was examined by qRT-PCR. The results showed a high abundance of expression in 7860 and $\mathrm{ACHN}$ cells $(\triangle \mathrm{Ct}<12$, Figure $3 \mathrm{~A})$. We examined the rate of pole2 knockdown following infected by shPole2 and shCtrl, and the relative mRNA expressions of pole2 were $0.302 \pm 0.032$ and $1.006 \pm 0.132$ in ACHN cells, respectively $(p=0.009)$. The knockdown rate of pole2 was $69.8 \%$. Knockdown of pole2 mRNA expression in 7860 cells was also successfully performed using the same way. The relative expression of pole2 mRNA was reduced to $0.197 \pm 0.016$, and the knockdown rate was $80.3 \%(p=0.000)$ (Figure 3B and 3C).

\section{Pole2 promotes proliferation of renal cancer cells in vitro}

To investigate the biological role of pole2 in renal cancer cells, a lentivirus-mediated short hairpin RNA (shRNA) was used to knockdown pole2 expression in 7860 and ACHN cells. The flurescent images were captured using the Celigo Cell Counting, and the results of cell counting revealed that the proliferation of pole2-knockdown 7860 cells was significantly inhibited, compared with control cells, the finding was further confirmed in ACHN cells (Figure 4A). Importantly, the MTT assay also confirmed that the proliferation of pole2-knockdown 7860 cells and ACHN cells were lower than the control cells group ( $p<$ 0.05). The result indicated that the proliferation of 7860 cells was reduced after knockdown pole2 (Figure $4 B$ and $C)$.

\section{Pole2 knockdown induce cell cycle S phase arrest in RCC cells}

We next evaluated the mechanisms responsible for the anti-proliferation effect of the pole2 knockdown, its role on the cell cycle progression of 7860 and $\mathrm{ACHN}$ cells. Compared with the corresponding control cells, the percentages of cells at the $S$ phase in 7860 cells(Figure $5 \mathrm{~A}$ and $\mathrm{C}$ ) and ACHN cells (Figure 5B and D) were significantly increased (in 7860 cells 28 vs $51 \%, p<0.05$; in ACHN cells 12.07 vs $33.15 \%$, $p<$ $0.05)$ ), on the contrary, the percentages of cells at the $\mathrm{G} 1$ phase were clearly reduced (in 7860 cells 43.29 vs $37.63 \%, p<0.05$; in ACHN cells 60.40 vs $41.59 \%, p<0.05)$. Taken together, pole2-knockdown would result in cell cycle arrest at the $S$ phase in renal cancer cells.

\section{Pole2 inhibits the apoptosis of renal cancer cells}

we evaluated the role of pole2-knockdown on the apoptosis of renal cancer cells. The results showed that the apoptosis of pole2-knockdown 7860 cells and ACHN cells was significantly promoted, comparing to those in the control cells $(p<0.05)$ (Figure 6A, 6B and 6C). To further confirm the role of pole2 on the apoptosis, we examined the activity of caspase3/7. Similarly, comparing with control cells, the activity of caspase $3 / 7$ showed higher in the pole2-knockdown 7860 cells and ACHN cells $(p<0.05)$ (Figure 6D). 


\section{Pole2 knockdown inhibited AKT/mTOR signaling pathway in ACHN cells}

On the based above mentioned role of pole2 on renal cancer cells, we next examined the mechanism underlying the effect of pole2 knockdown on related downstream proteins. Comparing with control cells group, western blot analysis revealed that phosphorylated mTOR (p-mTOR) was significantly reduced in pole2-knockdown cells group $(p<0.05)$, we also observed that mTOR expression was also downregulated, but it did not reach statistical difference. Meanwhile, phosphorylated AKT ( $p$-AKT) expression also showed a significant decrease $(p<0.05)$, whereas, we did not find a significant difference in the expressions of AKT (Figure 7A and 7B). These results suggested that down-regulation of p-mTOR and $p$ AKT expression by knockdown of pole2 may be possible mechanism of effect of pole2 on renal cancer cell proliferation, apoptosis and cell cycle.

\section{Discussion}

Our study, for the first time, revealed that pole2 regulates proliferation, apoptosis and cell cycle of renal cancer cells. Moreover, data analysis of $96 \mathrm{ccRCC}$ patients with follow-up information indicated that expression level of pole2 is positively related to Fuhrman grade of ccRCC, additionally, pole2-high patients with ccRCC have shorter overall survival than pole2 low patients. Taken together, these findings firmly suggest that pole2 may be an important mediator in ccRCC progression and one of the prognosis factors.

Pole2 is a 59-KDa protein, which plays multiple biological roles in eukaryotic cells, and pole2 mutation would result in some diseases, such as immunodeficiency, colorectal carcinoma and so on. Pole2 is DNA polymerase B subunit gene, which is one of the central components in DNA replication, repair, recombination and cell cycle control. The transcriptional control and expression of pole2 was regulated by two E2F-pocket protein complexes, one associated with Sp1 and the other with NF-1. ${ }^{10}$ According to the previous report, DNA replication stress is considered a common phenomenon across human malignancies, which may be induced by oncogene or the loss of tumor suppressors, ${ }^{11}$ especially, the inactivation of the tumor-suppressor von Hippel-Lindau and loss of heterozygosity (LOH) of chromosome $3 p$ are the most frequent genomic aberrations, ${ }^{12,13}$ which may contribute to the pathogenesis and progress in CCRCC. In our study, based on the results of immunohistochemistric examination, we found that pole2 expression was upregulated in the high Fuhrman grade (G3) ccRCC histological sections, compared with that in the lower grade (G1-G2), while similar tendency does not find in sections of different $T$ stage, the results may be correlated with its basic function that pole2 involves in DNA repair and in chromosomal DNA replication, which were a indicator of cancer aggressiveness, ${ }^{14,15}$ comparing with T stage based on the tumor size, indoubtly, Fuhrman grade is more used to evaluate kidney tumor aggressiveness, ${ }^{16,17}$ so it also implicated that pole2 expression should be an indirect indicator kidney tumor aggressiveness. In addition, we also found in that the number of positive cells in sections of Fuhrman 4 and stage 4 were lower than sections of lower stage and grade 4 , the small proportion of 
patients with stage 4 and Fuhrman grade 4 may be the mainly reasons, but number of pole2-positive cells still tended toward to increase in stage 1 - 4 and from grade 1-3. Thus, pole2 may act as an oncogene in ccRCC. Because pole2 can increase the proliferation of ccRCC cells, but it inhibited the apoptosis of ccRCC cells.

Based on the above mentioned the functions of pole2 in RCC cells, we also investigated the potential mechanisms for underlying such activities. Our study indicated that pole2 may participate in regulation of AKT/mTOR signaling pathway, which is closely related to the progression and development of ccRCC. ${ }^{18}$ Pole2-knockdown renal cancer cells demonstrated a decrease of p-mMOR and p-AKT expression, compared with the control cells with pole2 overexpression, s moreover, given that pole2knockdown also can inhibit proliferation of RCC cells, and pole2 is positively related to the Fuhrman grade. These findings indicate that pole2 play a role in RCC may via regulating AKT/mTOR pathway, specially, it may increase phosphorylated level of AKT and mTOR, since some studies demonstrated that increased phosphorylated mTOR and AKT were closely related with aggressiveness and poor prognosis in patients with RCC, ${ }^{19,20}$ but due to the complex mechanisms underlying the progression and development of tumor, except for AKT/mTOR pathway, other pathways may involve in regulation of pole2.

Our study still has some limitations. The impact of pole2 on migration of RCC cells had not investigated, moreover, the sample of the metastatic ccRCC have not been included in the study, so it is unclear for relationship between pole2 and metastasis of cCRCC, the further study on the aspect is necessary. The small sample size and retrospective nature of the study are another limitation, to support the role of pole2 in the prognosis and treatment in patients with ccRCC, more samples and the prospective investigation is needed in the near future. Thirdly, although we presented a lot of data with regarding the role of pole2 in vitro, but the study is absent in vivo evaluation, which would be valuable for its potential clinical application.

\section{Conclusion}

Our study found the impact of pole2 on proliferation, apoptosis, and cell cycle of RCC cells by downregulating pole2 expression, and explored the mechanism responsible for its role. We also revealed association of pole2 expression with overall survival, TNM stage and Fuhrman grade in patients with ccRCC. These findings suggest that pole2 is a promising prognostic factor and therapeutic targeting for CCRCC.

\section{Abbreviations}

RCC: Renal cell carcinoma

mRCC: Metastatic renal cell cancer

ccRCC: Clear cell renal cell cancer 
VEGF: vascular endothelial growth factor

PBS: Phosphatebuffered saline

CT: Computed tomography

MRI: Magnetic Resonance Imaging

POLE2: DNA polymerase epsilon subunit 2

\section{Declarations}

\section{Ethics approval and consent to participate}

As for all the patients, written informed consents were obtained, and the protocol was approved by Ethical Committee of Harbin Medical University Cancer Hospital.

\section{Data Availability Statement}

All data included in this study are available upon request by contact with the corresponding author.

\section{Competing interests}

All of authors in the study have no conflict of interest to disclose.

\section{Funding}

This study was partly supported by Natural Scientific Research Project (H2018048) of Heilongjiang province Science and Technology Department, and it was partly supported by the China Scholarship Council.

\section{Authors' contributions}

S.Y. organized the study. W.L and L.K. analyzed the part data independently and presented the same results. L.K. and L.D. were responsible for the follow-up of ccRCC patients; W.W. and P.H. were responsible for immunohistochemistric staining and analysis. L.C. and X.Y. were responsible for collection of samples and data in the hospital. C.Y. and C.Y. were responsible for statistical analysis. T.L. conceived and wrote the manuscript.

\section{Acknowledgement}


We gratefully appreciated support in technique from Professor Cheng Zheng and Dr. Cai (First affiliated Hospital of Harbin Medical University, Harbin, Helongjiang, China).

\section{References}

1. Chow WH, Devesa SS, Warren JL, et al. Rising incidence of renal cell cancer in the United States. JAMA. 1999; 281:1628-1631.

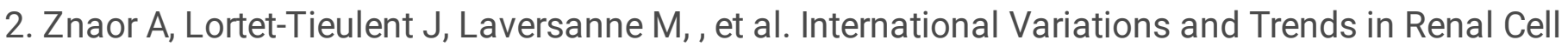
Carcinoma Incidenceand Mortality. Eur Urol. 2015; 67: 519-30.

3. Patel C, Ahmed A, Ellsworth P. Renal cell carcinoma: a reappraisal. Urol Nurs. 2012; 32: 182-190.

4. Saito K, Kihara K. Role of C-reactive protein as a biomarker for renal cell carcinoma. Expert Rev Anticancer Ther. 2010; 10: 1979-1989.

5. Modi PK, Farber NJ, Singer EA. Precision oncology: identifying predictive biomarkers for the treatment of metastatic renal cell carcinoma. Transl Cancer Res. 2016; 5: S76-80.

6. Callén E, Nussenzweig MC, Nussenzweig A. Breaking down cell cycle checkpoints and DNA repair during antigen receptor gene assembly. 2007; 26: 7759-64.

7. Frugoni F, Dobbs K, Felgentreff K, et al. A novel mutation in the POLE2 gene causing combined immunodeficiency. J Allergy Clin Immunol. 2016; 137: 635-638.

8. Spier I, Holzapfel S, Altmüller J, et al. Frequency and phenotypic spectrum of germline mutations in POLE and seven other polymerase genes in 266 patients with colorectal adenomas and carcinomas. Int J Cancer. 2015; 137: 320-31.

9. Wang H, Liu C, Han J, et al. HER2 expression inrenal cell carcinomais rare and negatively correlated with that in normal renal tissue. Oncol Lett. 2012; 4: 194-198.

10. Huang D, Jokela M, Tuusa J, et al. E2F mediates induction of the Sp1-controlled promoter of the human DNA polymerase epsilon B-subunit gene POLE2. Nucleic Acids Res. 2001; 29: 2810-21.

11. Halazonetis TD, Gorgoulis VG, Bartek J. An oncogene-induced DNA damage model for cancer development. 2008; 319: 1352-1355.

12. Dalgliesh GL, Furge K, Greenman C, et al. Systematic sequencing of renal carcinoma reveals inactivation of histone modifying genes. 2010; 463: 360-363.

13. Larkin J, Goh XY, Vetter M, Pickering L, et al. Epigenetic regulation in RCC: opportunities for therapeutic intervention? Nat Rev Urol. 2012; 9: 147-155.

14. Na R, Wu Y, Jiang G, et al. Germline mutations in DNA repair genes are associated with bladder cancer risk and unfavourable prognosis. BJU Int 122, 808-813 (2018).

15. Parker AS, Eckel-Passow JE, Serie D, Hilton T, Parasramka M \& Joseph RW et al. Higher expression of topoisomerase II alpha is an independent marker of increased risk of cancer-specific death in patients with clear cell renal cell carcinoma. Eur Urol 66, 929-35 (2014).

16. Kim H, Inomoto C, Uchida T, Furuya H, Komiyama T \& Kajiwara H et al. Verification of the International Society of Urological Pathology recommendations in Japanese patients with clear cell 
renal cell carcinoma. Int J Oncol. 2018; 52: 1139-1148.

17. Andreiana BC, Stepan AE, Mărgăritescu C, et al. Histopathological Prognostic Factors in Clear Cell Renal Cell Carcinoma. Curr Health Sci J. 2018; 44: 201-205.

18. Ribback S, Cigliano A, Kroeger N, et al. PI3K/AKT/mTOR pathway plays a major pathogenetic role in glycogen accumulation and tumor development in renal distal tubules of rats and men. 2015; 6:13036-48.

19. Rausch S, Schollenberger D, Hennenlotter J, Stühler V, Kruck S \& Stenzl A et al. mTOR and mTOR phosphorylation status in primary and metastatic renal cell carcinoma tissue: differential expression and clinical relevance. J Cancer Res Clin Oncol. 2019; 145: 153-163.

20. Mizuno T, Kamai T, Abe $\mathrm{H}$, et al. Clinically significant association between the maximum standardized uptake value on 18F-FDG PET and expression of phosphorylated Akt and S6 kinase for prediction of the biological characteristics of renal cell cancer. BMC Cancer. 2015; 15: 1097.

\section{Figures}

A

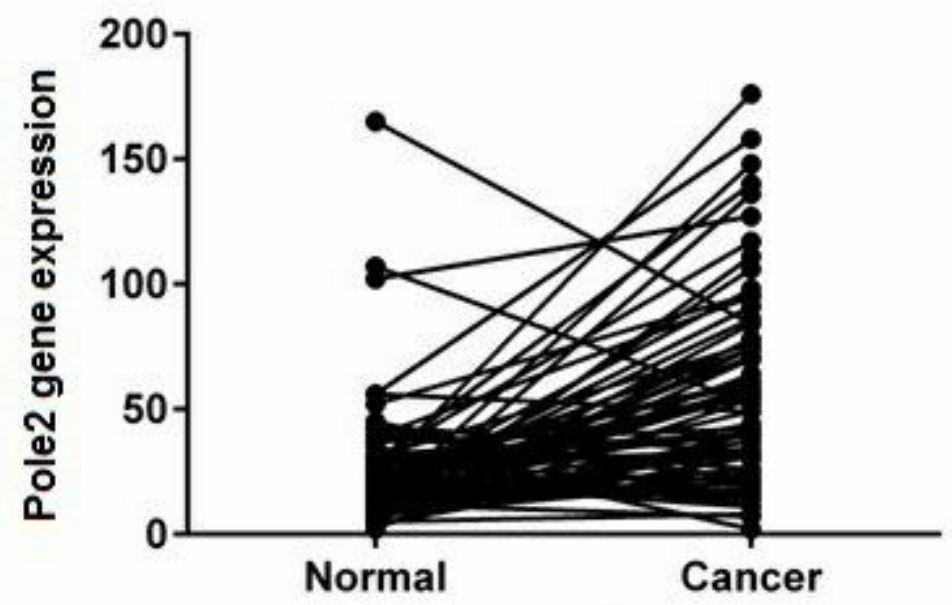

B

Adjacent normal tissue

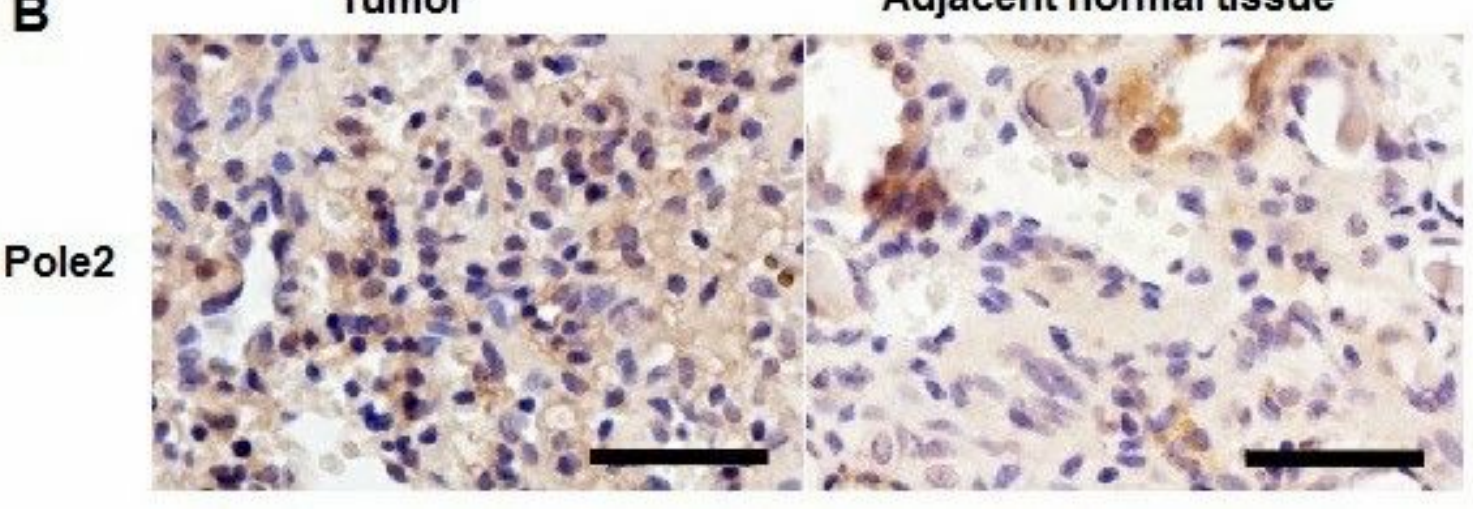

Figure 1

The increased pole2 expression in RCC tissues. A. compared with adjacent normal kidney tissues, pole2 gene expression was significantly up-regulated in cancerous tissues $(p<0.01)$. B. representative pole2 
staining images in RCC section and non-cancerous section were shown.

A

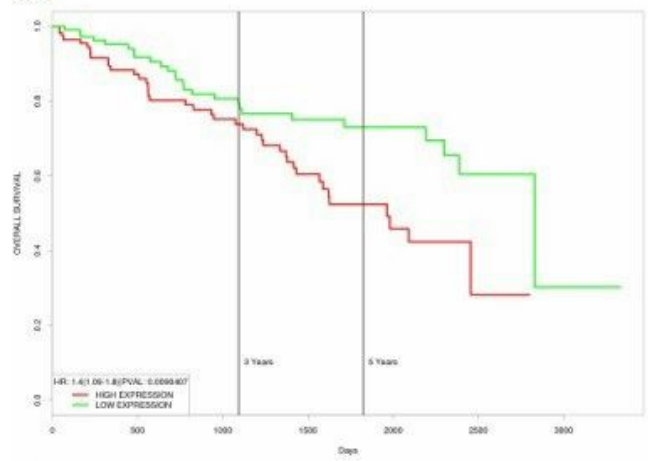

B

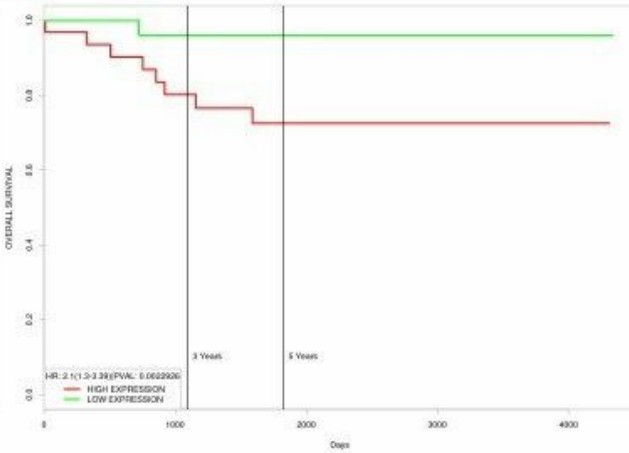

C

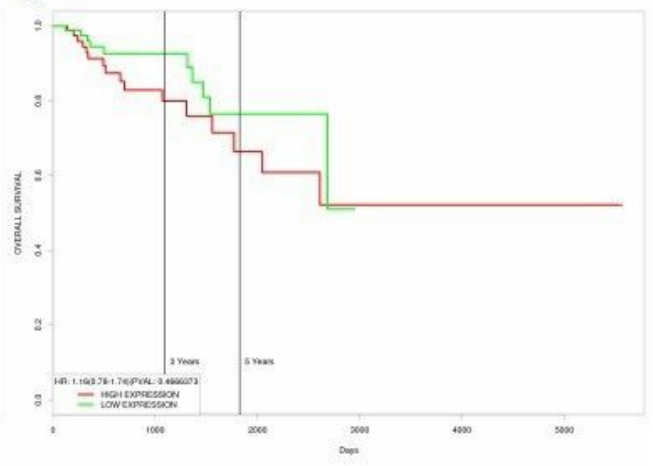

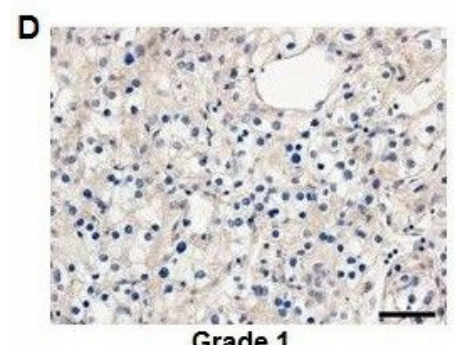

Grade 1

E

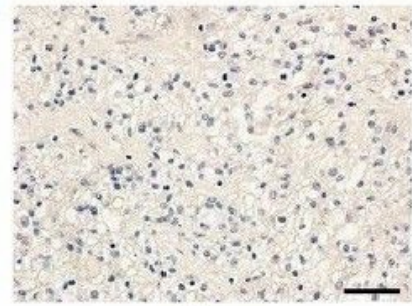

T1

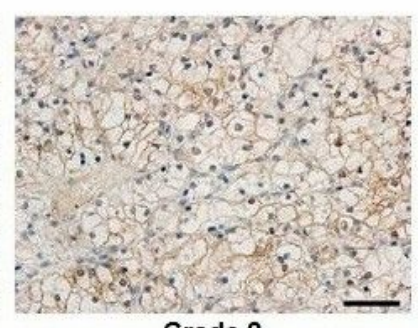

Grade 2

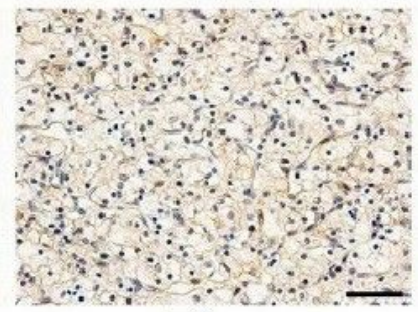

T2

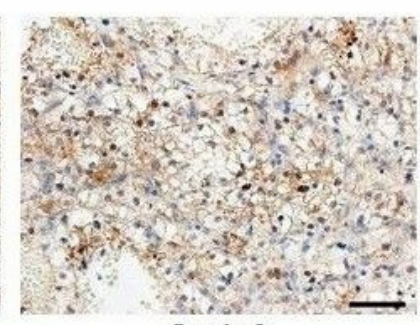

Grade 3

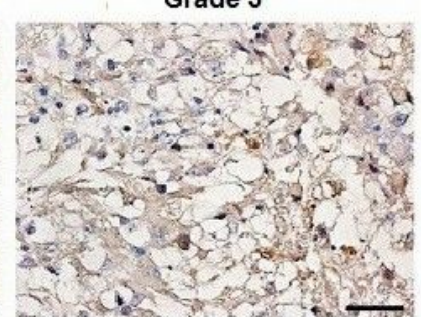

T3

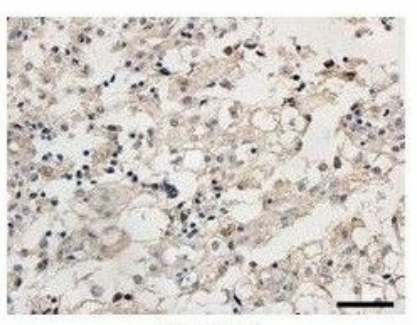

Grade 4

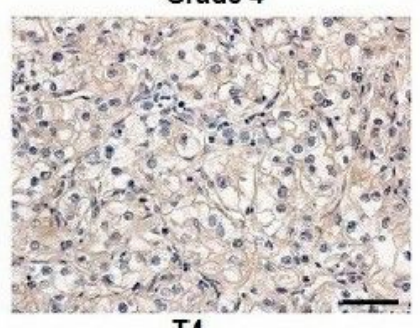

T4

Figure 2

Knockdown of pole2 gene inhibit proliferation of 7860 and ACHN cells. A. fluorescence microscope showed that 7860 cells infected shPole2 (lower panel) was growing slower than cells infected shCtrl (green staining) from day 1 to day 5. B. and C. Celigo cell counting indicated that the cells infected shPole2 did not significantly increase, comparing with cells infected shTtrl $(p<0.01)$. D. and E. OD and fold values of 7860 and ACHN cells infected shPole2 and shTrol were shown based on the result of MTT assay. ${ }^{*}<0.05$, compared with cells infected shTrol. 
A
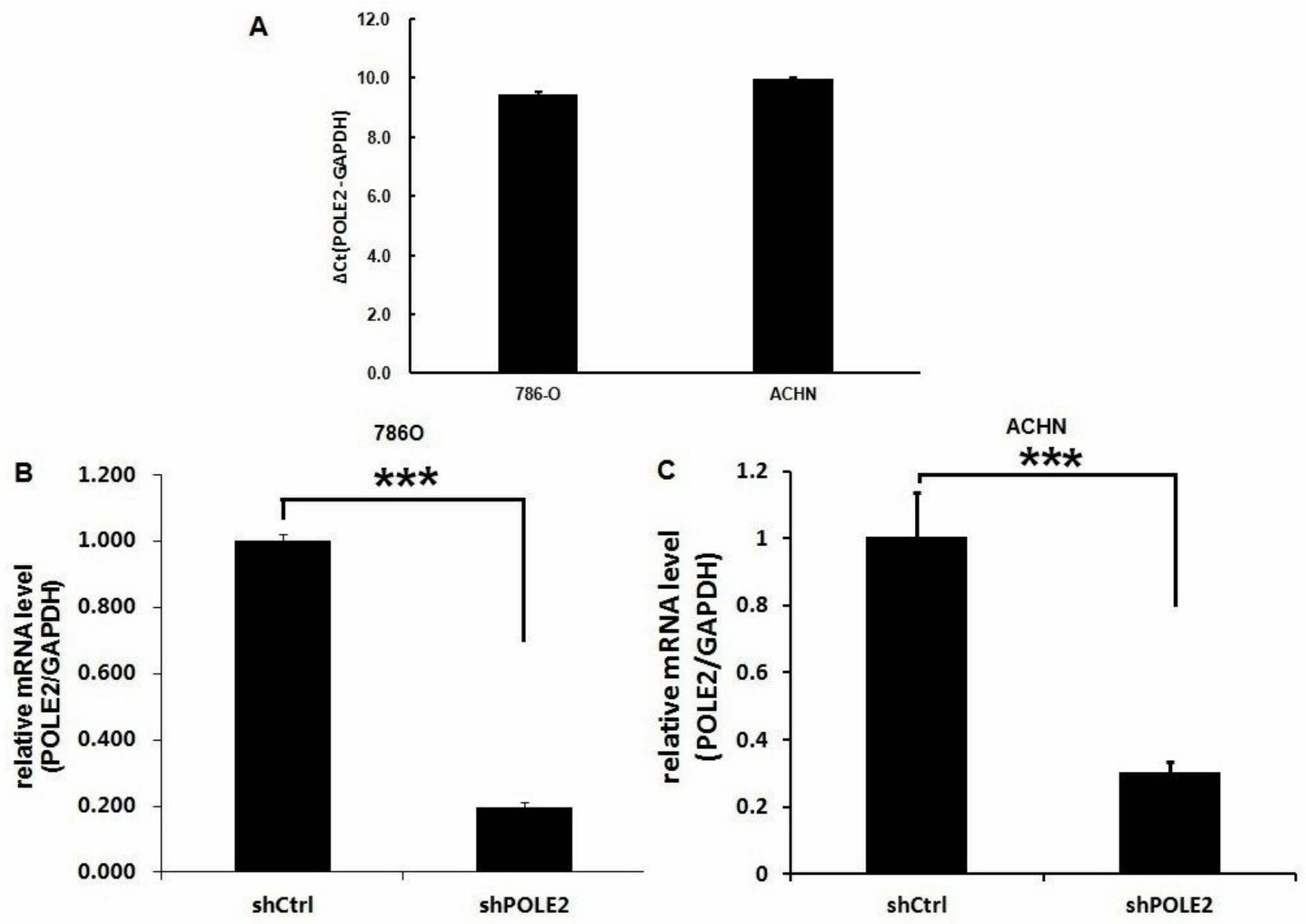

\section{Figure 3}

Apoptosis of 7860 and ACHN cells was promoted by infected shPole2 using flow cytometry and measuring caspase3/7 activity. A. and B. show representative flow cytometric images for 7860 and ACHN cells, respectively. C. The increased percentages of cell apoptosis in 7860 and ACHN cells infected shPole2 were shown, respectively. D. Caspase $3 / 7$ activity was significantly increased in 7860 and ACHN cells infected shPole2, respectively. $* \star<0.01$. 
A

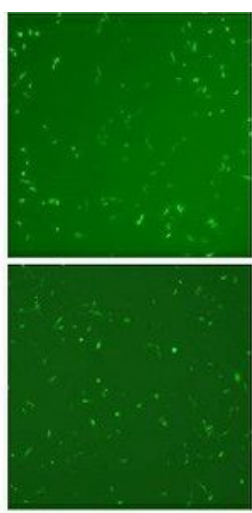

Day 1

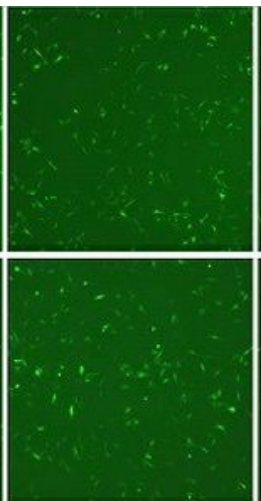

Day 2
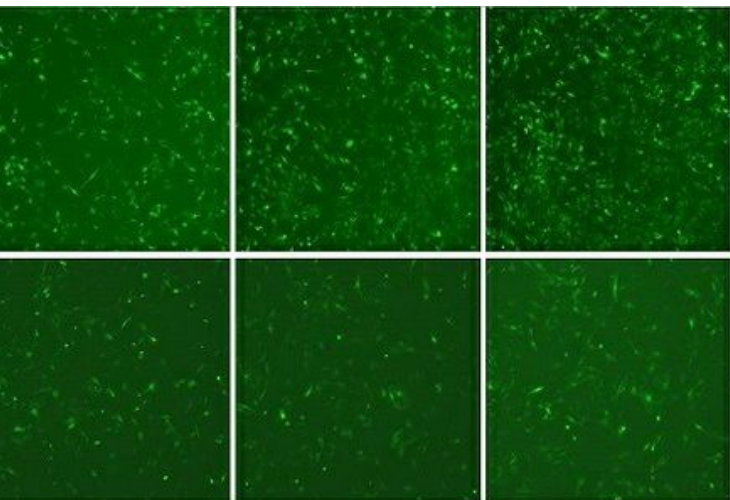

shCtrl
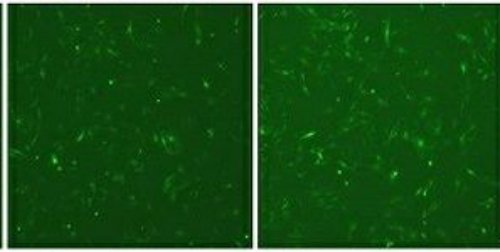

ShPOLE2
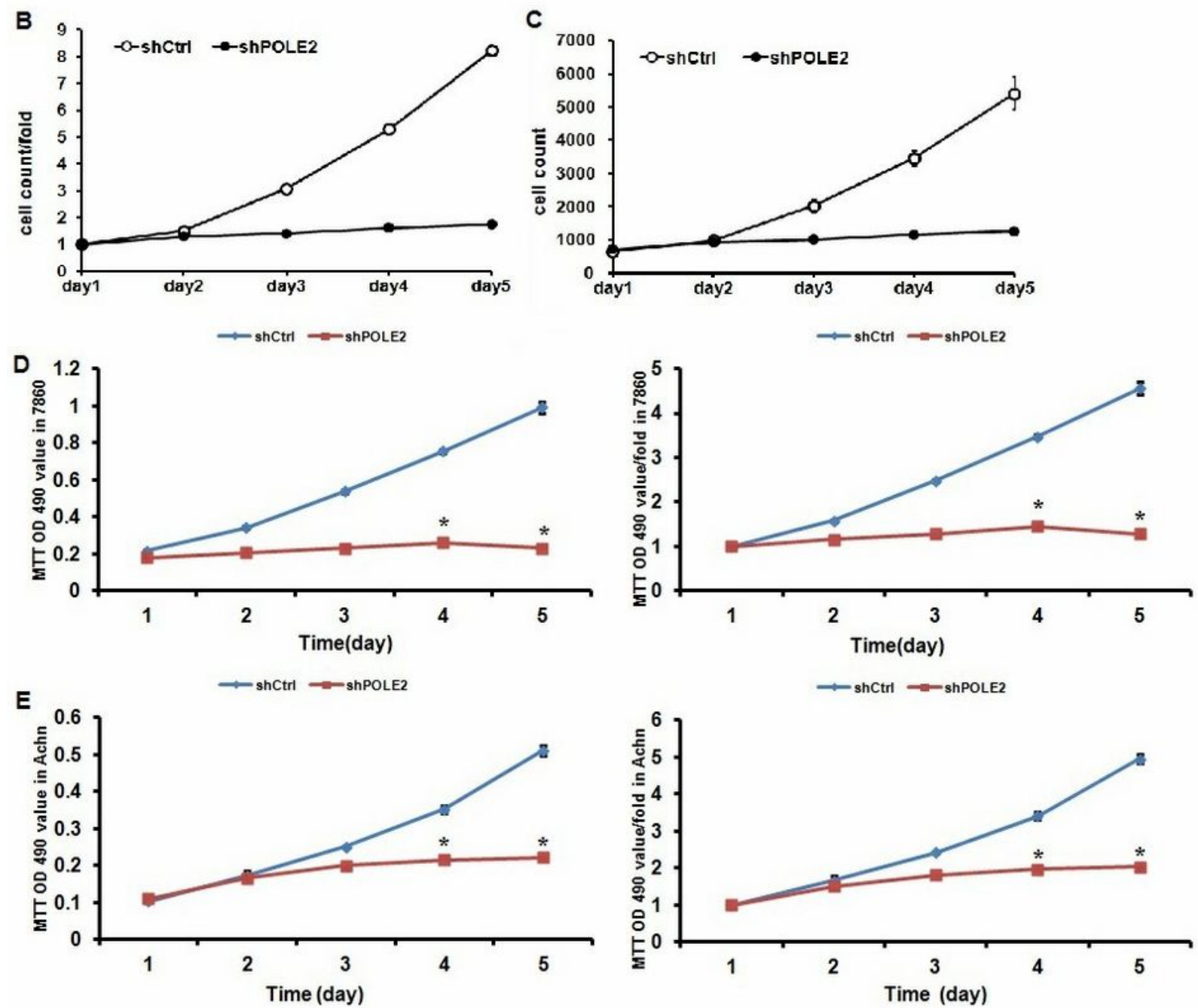

Figure 4

Cell cycle distribution was analyzed by flow cytometry at day 5 after infected lentivirus. A. and B. One representative experiment in 7860 and $A C H N$ cells were shown, respectively. C. and D. Proportion of 7860 and ACHN cells in different phases of cell cycle were shown. $* \star<0.01$. 

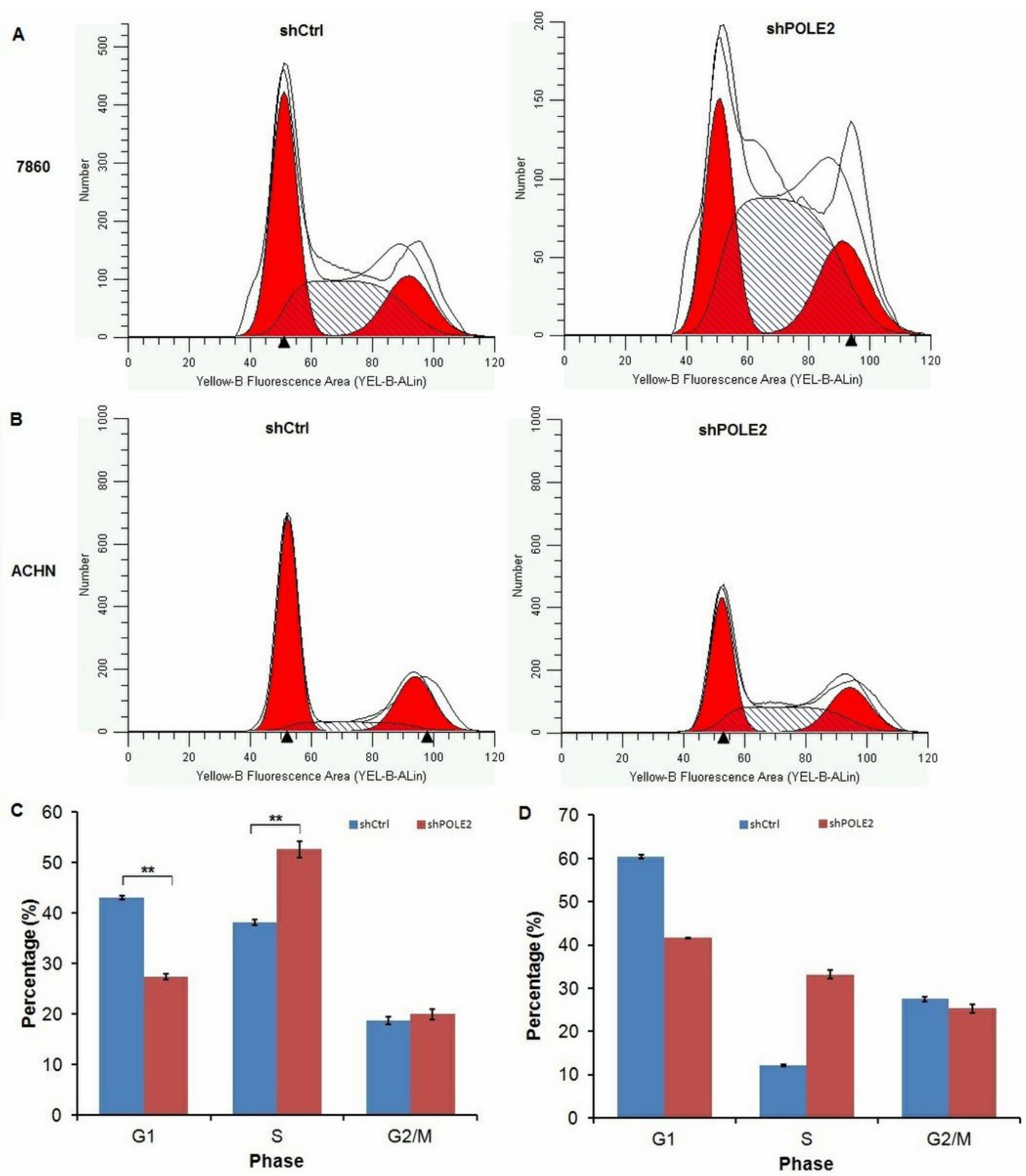

\section{Figure 5}

Kaplan-Meier survival curves of patients with clear cell RCC (A), chromophobe RCC (B) and papillary RCC (C) in different levels of pole2 expression. Overall survival of patients with clear cell RCC. D. and E. Representative immunohistochemistric staining of pole2 in different Fuhrman grades and stages (Original $\times 400$ ), and brown- color nuclear staining represent positive results, which were showing 
increased a tendency in the ways of grade and stage dependent. F and G. Immunohistochemical analysis of pole2-positive cells in clear cell RCC tissues with different stage and grade. ${ }^{*}<0.05$.

A
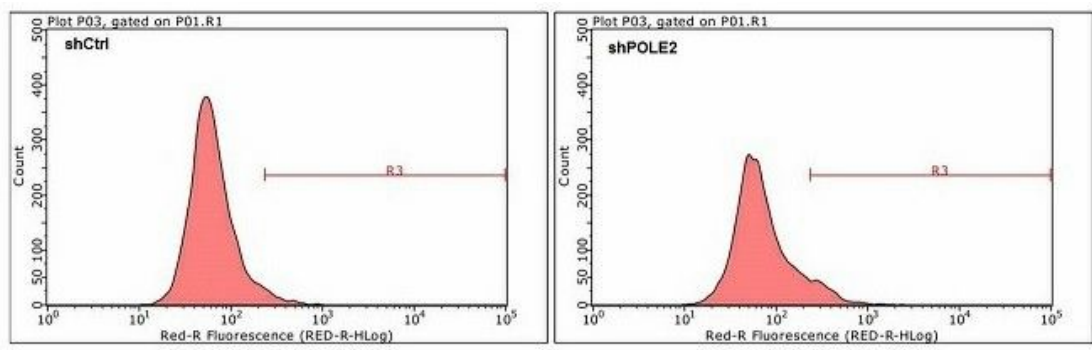

B
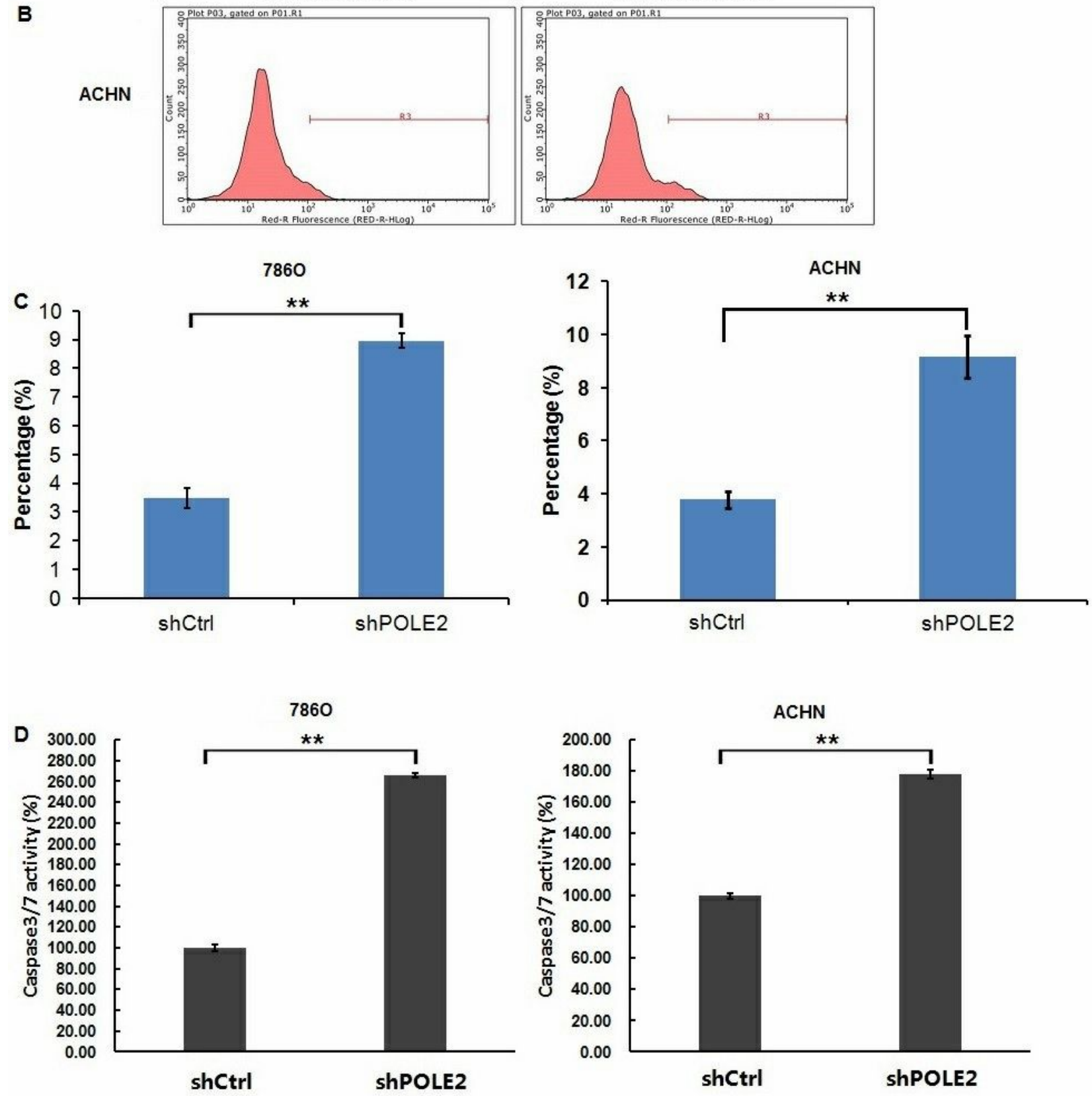

Figure 6

Pole2 mRNA expression in 7860 and ACHN cells and knockdown rate of pole2 by quantitative real time PCR. A. High abundance of pole2 mRNA in 7860 and ACHN cells. $\triangle \mathrm{Ct}$ is equal to the difference between 
the $\triangle$ Ct value of pole2 and GAPDH. B. Knockdown rate of pole2 in 7860 cells. C. Knockdown rate of pole2 in ACHN cells. *** $<0.001$.
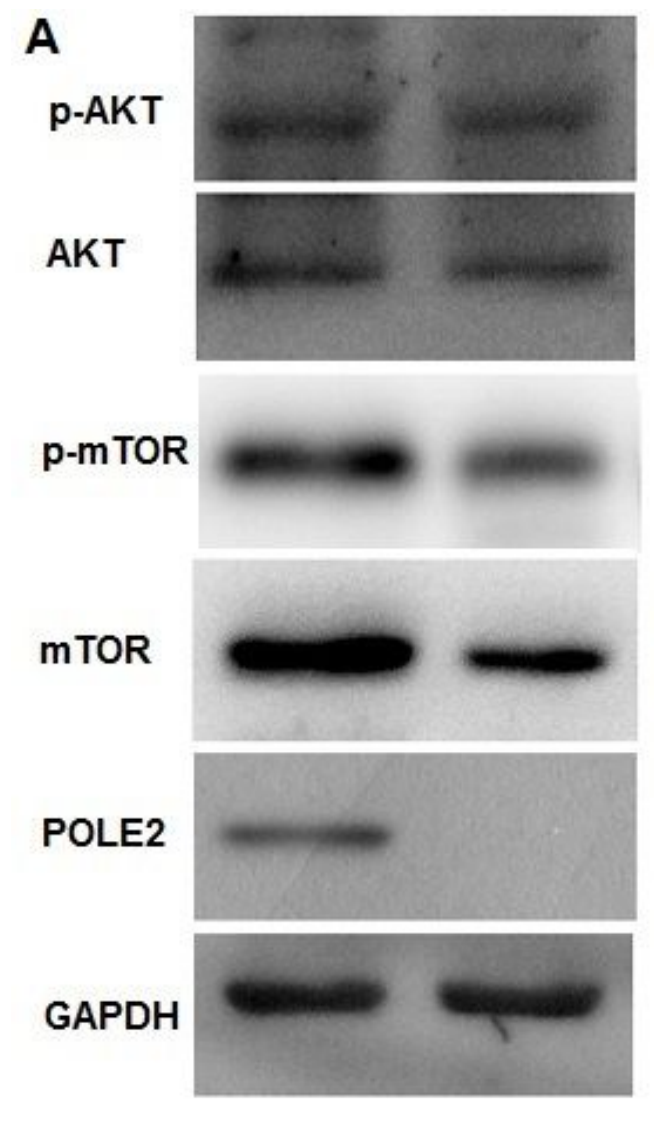

shCTRL ShPOLE2

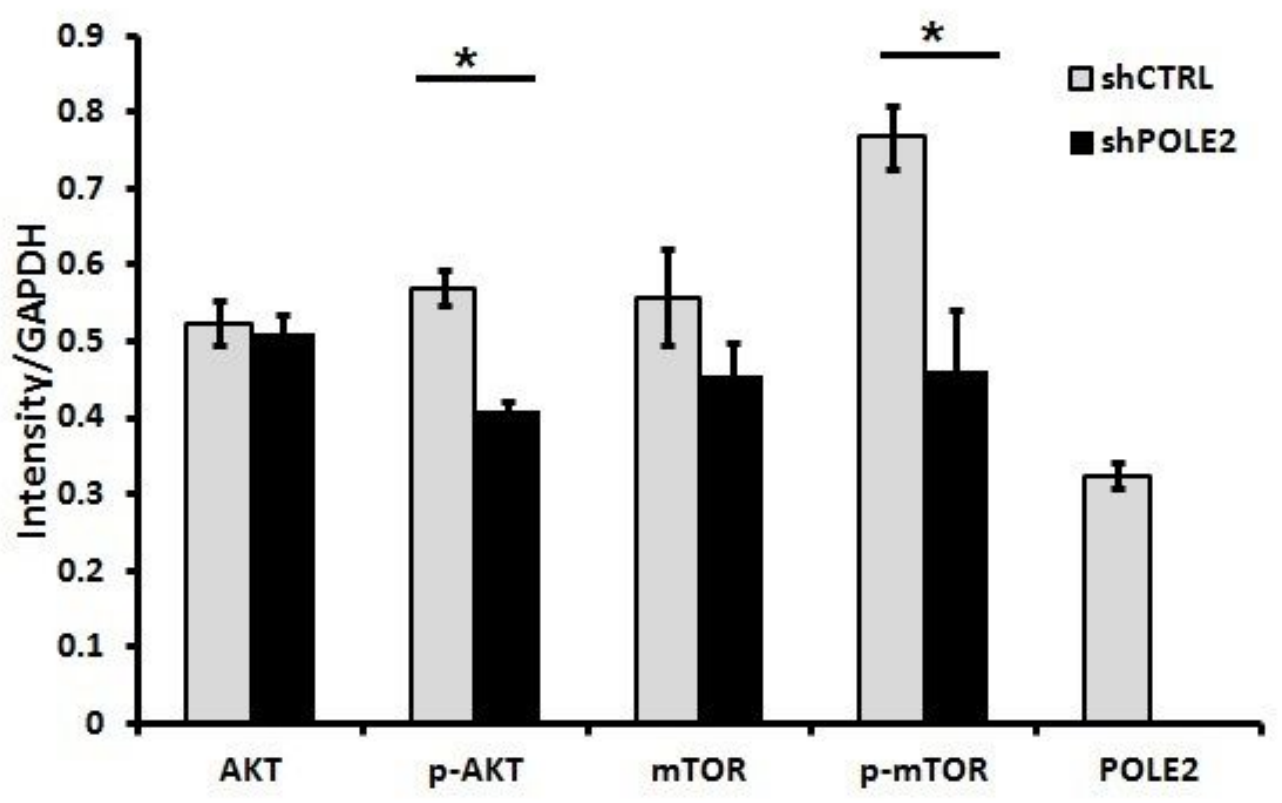

Figure 7

Knockdown of pole2 suppressed AKT/mTOR signaling pathway. A and B. ACHN cells were infected with shPOLE2 and shCTRL lentivirus, respectively. The levels of AKT, p-AKT, mTOR and p-mTOR proteins were analyzed by western blot. * $<0.05$. 